\title{
HeREDitary Stomatocytosis: an UNUSUAL CAUSE of SEVERE NEONATAL JAUNdice
}

Singapore Med J 2018; 59(9): 505 https://doi.org/10.11622/smedj.2018115

Dear Sir,

Haemolysis should be suspected when a newborn presents with early or excessive jaundice. ${ }^{(1,2)}$ Classical haemolytic disease of the newborn that is associated with maternofetal blood group incompatibility is still the most common cause of haemolytic neonatal jaundice (NNJ) worldwide. Intrinsic erythrocytic disorders, such as glucose-6-phosphate dehydrogenase (G6PD) deficiency, are important causes of NNJ in malaria-endemic regions. Hereditary spherocytosis is an important differential diagnosis when no immediate cause for NNJ is found. Hereditary stomatocytosis, another erythrocytic membranopathy, has rarely been associated with severe NNJ. Two cases are described below.

Case 1 is a full-term female infant who was admitted on Day 10 of life with a serum bilirubin level of $326 \mu$ mol/L. Her haemoglobin level was $10.3 \mathrm{~g} / \mathrm{dL}$. Direct antiglobulin test and G6PD deficiency screen were negative. Findings on the peripheral blood film were consistent with stomatocytosis (Fig. 1a). The jaundice responded to phototherapy and the haemoglobin level dropped to $7.2 \mathrm{~g} / \mathrm{dL}$. She recovered after a red blood cell transfusion. The child's father was found to have the same condition.

Case 2 involves a full-term male infant who was noted to have severe jaundice on Day 3. His serum bilirubin and haemoglobin levels were $345 \mu \mathrm{mol} / \mathrm{L}$ and $12.4 \mathrm{~g} / \mathrm{dL}$, respectively. Direct anti-globulin test and G6PD deficiency screen were negative. He received red blood cell transfusion on Day 9 and the haemoglobin level dropped to $8.0 \mathrm{~g} / \mathrm{dL}$. Haematological consultation was made, but the blood smears done prior to the transfusion were not available. Review of the family history revealed that the child's mother was anaemic during her pregnancy. Coombs-negative autoimmune haemolytic anaemia was diagnosed. Prednisolone was administered but her haemoglobin level remained at 8-9 g/dL. This prompted a blood film examination (Fig. 1b), which confirmed a diagnosis of stomatocytosis in both the mother and child. The infant required weekly red blood cell transfusion until the second month of life. Thereafter, he recovered spontaneously, and his unsupported haemoglobin level at five months was $13.2 \mathrm{~g} / \mathrm{dL}$.

Hereditary stomatocytosis is a group of autosomal-dominant inherited blood diseases in which 'leaky' erythrocytes are dehydrated or rarely overhydrated. The affected red blood cells assume a cup-shaped morphology and their central pallor turns into a slit-like appearance on the blood film, earning their descriptive title. As a whole, adults with hereditary stomatocytosis are mildly symptomatic. ${ }^{(3)}$ Severe NNJ associated with stomatocytosis has only been reported once. ${ }^{(4)}$ The discovery of PIEZO1, a mechanosensitive ion channel protein, has revolutionised our understanding of erythrocytic membranopathies. Different mutations in the PIEZO1 gene account for the various manifestations, including anaemia, gallstones, pseudohyperkalaemia, transient neonatal lymphoedema and hydrops. ${ }^{(5)}$ As specific mechanical and genetic studies on erythrocytes are still only restricted to a few research laboratories, the diagnosis of hereditary stomatocytosis remains clinical. It should be included in the differential diagnosis of pathological NNJ. The importance of blood film examination cannot be overemphasised.
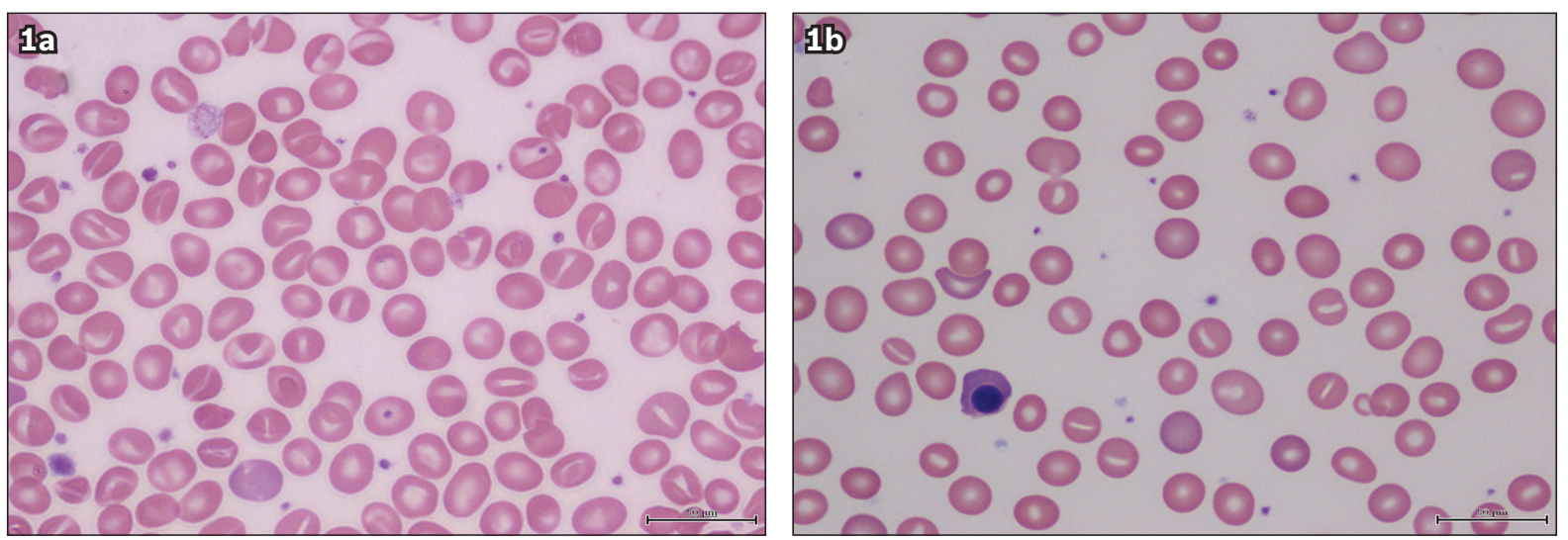

Fig. 1 Photomicrographs of the peripheral blood smear of (a) the infant in Case 1 and (b) the mother in Case 2 show abundant stomatocytes with a slit-like central pallor (Giemsa stain, × 100).

Yours sincerely,

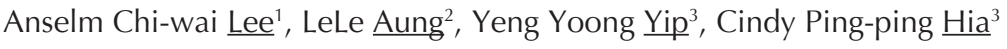

${ }^{1}$ Children's Haematology and Cancer Centre, Mount Elizabeth Hospital, ${ }^{2}$ Parkway Cancer Centre, Gleneagles Hospital, 3International Child and Adolescent Clinic, Singapore. anselm.cw.lee@gmail.com

\section{REFERENCES}

1. National Collaborating Centre for Women's and Children's Health. Neonatal Jaundice. Royal College of Obstetricians and Gynaecologists: London, 2010.

2. Christensen RD, Nussenzveig RH, Yaish HM, et al. Causes of hemolysis in neonates with extreme hyperbilirubinemia. J Perinatol 2014; 34:616-9.

3. Da Costa L, Galimand J, Fenneteau O, Mohandas N. Hereditary spherocytosis, elliptocytosis, and other red cell membrane disorders. Blood Rev 2013; 27:167-78.

4. Huppi PS, Ott P, Amato M, Schneider H. Congenital haemolytic anaemia in a low birth weight infant due to congenital stomatocytosis. Eur J Haematol 1991; 47:1-9.

5. Andolfo I, Alper SL, De Francischi L, et al. Multiple clinical forms of dehydrated hereditary stomatocytosis arise from mutations in PIEZO1. Blood 2013; 121:3925-35. 